\title{
Las tecnologías de información y comunicación en el ámbito sanitario
}

\author{
Marisa Correcher Palau \\ Coordinadora de los Sistemas de Información Asistenciales \\ del Departamento de Salud del Hospital de La Fe
}

correcher_mar@gva.es

\begin{abstract}
Resumen
El presente artículo aborda la situación de los sistemas de información en el ámbito sanitario y con el condicionante actual de la grave pandemia del COVID 19. Partiendo de la situación en la que nos encontramos en septiembre de 2021 incorporando los cambios acontecidos en el campo de la informática, e imaginando como van a cambiar algunos aspectos de las tecnologías de la información. Nuevas formas de hacer aplicaciones sanitarias, generalización del uso de las tecnologías para el modelo asistencial, la información como palanca de cambio en la práctica clínica, incorporando a todo ello de manera trasversal las consideraciones de seguridad y privacidad de la información.
\end{abstract}

Analizaremos el estado del arte de los sistemas de información sanitarios desde el punto de vista de un departamento de salud con un hospital terciario. En él expondremos una visión de futuro que desvele los retos tecnológicos en el sector de la Salud.

\section{Introducción}

En todos los sectores, la pandemia ha sido un punto de inflexión en la evolución de la tecnología, pero es en el sector sanitario si cabe donde este cambio ha sido mayor debido a la "crisis sanitaria" que ha tensado todos los cimientos del sector, y por consiguiente a cuestionado su "madurez tecnológica".

Esta tensión ha sacado a flote lo mejor y lo peor de la organización sanitaria, sus procesos y los sistemas que le dan cobertura. Llevamos mucho tiempo hablando de bigdata, de telemedicina, de medicina de precisión, de tratamientos personalizados, y otros conceptos que definen las nuevas prácticas clínicas que se van incorporando, pero que se van desarrollando a un ritmo lento e incorporando en pequeñas dosis a la operación diaria del negocio. La realidad, es que nuestros sistemas son eminentemente presenciales, mucho más manuales de lo que nos gustaría y muy poco personalizados en general. 
Los retos que hemos abordado en el último año y medio han sido enormes en todas las capas de la asistencia sanitaria: la salud pública, la atención primaria, la atención hospitalaria y la medicina preventiva.

A pesar de todo, en estos meses, los servicios informáticos de la sanidad pública, con la inestimable colaboración del sector privado, hemos sido capaces de implementar aplicaciones y herramientas tecnológicas encaminadas a organizar y agilizar la labor de todos los profesionales del sistema de salud, y se han desplegado las infraestructuras de comunicaciones para que todos los sistemas estuvieran en funcionamiento con todas las capacidades necesarias.

En los momentos más duros de la pandemia, en la Comunidad Valenciana, se han tenido que arreglar, aumentar, mejorar y modernizar sistemas de información, algunos con más de 30 años de existencia y todo en un tiempo récord. Esto ha supuesto que el sector sanidad ha forzado un salto "cuántico" en la implantación de las tecnologías en el momento más hostil posible, demostrando nuestra capacidad de adaptación en todos los aspectos de la prestación sanitaria, incluyendo por supuesto el área tecnológica que es la que desarrollamos en este artículo.

No debe escapar al lector, que la tecnología no es el fin en sí, sino el medio que apoya a los fines de la organización sanitaria, que son la prestación asistencial, la investigación y la formación en ciencias de la salud. Así, los responsables de los servicios sanitarios son conscientes de la importancia del uso de las tecnologías de la información para dar soporte a la mayoría de los actos clínicos asistenciales, a la investigación en sanidad y a la formación de futuros profesionales; y sustentando la toma de decisiones tanto clínicas como las que atañen a la gestión de los recursos.

Por último, la dependencia que tienen las operaciones del sector salud en la tecnología de la información, nos hacen poner el foco en los riesgos que debemos prevenir para salvaguardar la confidencialidad, integridad y disponibilidad de estas tecnologías. Esta cuestión saltó del mero ámbito informático a los ámbitos de gobierno del sistema nacional de salud en mayo del 17, cuando el ransomware wannacry desplegó un ataque a escala mundial que afectó a más de 141.000 equipos informáticos, colapsando infraestructuras nacionales críticas como el servicio de salud británico, o las instalaciones en España de Telefónica, Iberdrola y Gas Natural.

A medida que avance en la lectura, el lector encontrará una reflexión sobre lo que hemos sido capaces de hacer, lo que no hemos logrado, y las lecciones que hemos aprendido para aplicarlas en el futuro. Aprendemos de los éxitos, pero sobre todo de los errores, y hemos de potenciar las tecnologías y procesos que han sido buenos para funcionar en el contexto de la pandemia y descartar las prácticas fallidas.

Como he dicho, tenemos que considerar varios aspectos para abordar el futuro del sector sanitario, y los desarrollaremos en estos puntos:

- Las aplicaciones sanitarias, presente y futuro.

- Nuevos modelos de atención sanitaria.

- La medicina moderna y la información para la toma de decisiones o para la investigación. 
- La ciberseguridad, privacidad de la información, y cumplimiento normativo.

Dándole un enfoque realista de la situación actual para hablar de aspectos de futuro, pero con la perspectiva de la situación real en la que estamos ubicados.

\section{Aplicaciones Sanitarias}

Abundando en la idea de que las aplicaciones sanitarias son básicas en la operación diaria del sector salud, ellas han sido una de las herramientas necesarias para combatir algunos aspectos de esta crisis sanitaria, dando respuesta a las necesidades que han irrumpido sin avisar en el Hospital. Cuando todo el país estaba en shock, allí estábamos los ingenieros informáticos y demás profesionales de la tecnología formando parte de la solución con nuestro trabajo.

Muchas de las deficiencias y las necesidades que hemos visto deberían convertirse en prioridades para el futuro, la integración entre sistemas y la capacidad de adaptación de los mismo son fundamentales. Tenemos que ver como conjugamos estas dos necesidades: integrar sistemas y flexibilizar los mantenimientos.

En el entorno sanitario valenciano estamos acostumbrados a dos tipos de software, uno engloba a las aplicaciones de desarrollo centralizado que tienen unos ciclos de producción absolutamente increíbles, se puede dar el caso de que una pequeña mejora que podría desarrollarse en pocos días, al entrar en los procesos de gestión de cambio de los grandes proyectos, puede tardar más de un año en que el profesional la tenga resuelta, justificándose en el gran número de solicitudes de cambio, en los tiempos de integración de los artefactos y en los correspondientes controles de calidad. En segundo lugar, tenemos las aplicaciones a medida que se hacen en cada departamento de salud y hospital por parte del propio personal informático, con un ciclo de desarrollo mucho más corto pero en general con un alto grado de acoplamiento con el Departamento que las desarrolla.

En el sector salud hay un piélago de aplicaciones que cubren todas sus áreas, pero especialmente es en el área de salud pública donde considero que menos se ha invertido y que tiene mayor necesidad de nuevos sistemas de información, por lo que debe ser una de las áreas donde más se potencie la inversión en tecnologías de la información en los próximos años. Si antes de la pandemia ya era una necesidad importante potenciar el uso de sistemas para recopilar y compartir la información relativa a la salud pública de los ciudadanos, ahora se ha convertido en un desarrollo imprescindible que permitirá tener la información epidemiológica a tiempo para dar respuesta a las necesidades de gestión de esta crisis y para poder abordar futuras pandemias o problemas generales de prevención de la salud de los ciudadanos.

El caso de la gestión del ciclo de vida de la administración de vacunas, desde que se adquiere hasta que se administra, es un ejemplo perfecto de lo que nos va a demandar el futuro. Necesitamos aplicaciones compartidas con capacidad de identificar a los ciudadanos en base a filtros (edad, enfermedad,...), que permitan acceder desde muchos centros y compartir la información con otros sistemas comunitarios, nacionales o europeos. Por poner un ejemplo hay que poner en valor el esfuerzo que ha realizado la administración sanitaria en la comunidad valenciana, su 
personal técnico y los partners tecnológicos, que han permitido en un tiempo record tener un documento consensuado con información para el "pasaporte covid", e integrarlo en los sistemas nacionales y europeos.

En el departamento de salud Valencia - La fe, para adaptarnos a las nuevas necesidades, hemos desarrollado e implantado nuevas prestaciones (citas telefónicas, envíos de notificaciones, comunicación de resultados negativos, ...), hemos modificado aplicaciones para incorporar nuevos circuitos asistenciales para dotarles de más información relacionada con la gestión de la pandemia (marcar pacientes covid, niveles de gravedad,...).

Por citar más ejemplos, partiendo del sistema de información operativo, se han desarrollado módulos y parametrizaciones para poder cumplir los requerimientos que se nos pedían en cada momento, y de un día para otro hemos tenido que dotar de sistema de información para una unidad de críticos en espacios que nos estaban preparados para ellos. O se ha habilitado una extensión en el sistema para un nuevo hospital de campaña, o se ha introducido en las nuestras aplicaciones la gestión de los "hoteles medicalizados".

La circunstancia nos ha forzado a una gestión ágil de los cambios en los sistemas de información, y en un tiempo récord hemos implementado "al vuelo" soluciones para adaptarnos a la nueva situación. Hemos hecho cambios impensables en un contexto normal, por ejemplo, la petición electrónica de pruebas de imagen, un proyecto acabado pero de escasa implantación por los usuarios, quienes seguían usando el modelo de papel, ha pasado a ser el método exclusivo con el que los servicios clínicos realizan las solicitudes al área de imagen. Ante la necesidad de reducir los contactos personales y el movimiento de personas, la resistencia al cambio desapareció y el proceso electrónico quedó implantado en apenas 15 días. La lección aprendida para el área que gestiona y toma las decisiones es que la organización está capacitada para la implantación de nuevos proyectos, y que la resistencia al cambio es mínima si hay un mandato claro.

La gran pregunta, ¿Como va a ser el futuro?: No lo sé, espero, quiero y deseo que podamos avanzar en metodologías de construcción y mantenimiento de software que permitan hacerlo más ágil en su mantenimiento y evolución, de más rápida implantación y sobre todo más cercano al usuario. Habrá que tener en cuenta que conviviremos con aplicaciones "antiguas" que seguirán siendo necesarias en la práctica clínica diaria hasta su sustitución. La complejidad de los sistemas de información en las grandes organizaciones lastra las posibilidades para rehacerlos desde cero, y la organización sanitaria valenciana es un claro ejemplo, podemos contrastar que cualquier avance que se acomete arrastra esa rémora que lo ralentiza.

Los analistas ya vislumbraban antes de la pandemia las tendencias que en 2020 se han manifestado imprescindibles. Yo veo de gran ayuda avanzar en portales de salud con aplicaciones orientadas al paciente, con capacidad de gestionar citas telefónicas o por vídeo conferencia, y con comunicación bidireccional que permita incorporar toda la información de su enfermedad, incluyendo la experiencia que aporte el propio paciente, la información directa de dispositivos clínicos conectados y la que recogen los sistemas hospitalarios y los propios profesionales en la historia clínica electrónica. 
Una gran debilidad del sistema es el tiempo que se puede dedicar en consulta a cada paciente, sobre todo en la atención primaria. No está en el ámbito de este artículo analizar las causas y las soluciones a esta debilidad, y no puedo más que animar a los responsables de la gestión del sistema valenciano de salud a que agudicen su ingenio en la búsqueda de soluciones que la resuelvan, y la tendencia se orienta hacia los portales de salud orientados al paciente, apoyados en chatbots, inteligencia artificial, algoritmos de recomendaciones clínicas mediante toma de decisiones complejas, siempre respaldado y avalado por un equipo facultativo cualificado y multidisciplinar. La falta de agilidad de la administración sanitaria del estado y de las comunidades autónomas no augura una rápida implantación de este tipo de soluciones en la sanidad pública, y creo que de momento este nicho de negocio lo ocuparán las empresas privadas sanitarias que ofrecerán modelos de atención muy dirigidos a la salud electrónica.

Esto no significa que estemos parados, la telemedicina es un importante reto que el sistema público está abordando, definiendo nuevos modelos asistenciales y proponiendo tecnologías. De los pilotos que venimos implantando en distintas etapas desde la apertura del nuevo hospital la Fe en 2011, las soluciones con mayor viso de realidad son las dirigidas a pacientes con necesidades de control hospitalarias que se puedan trasladar al domicilio, quedando para la próxima década los portales que faciliten la gestión de la promoción de la salud desde la atención primaria. En el siguiente apartado veremos cambios en el modelo que nos van a permitir desarrollar este tipo de atención.

Si nos centramos en el objetivo de la mejora de procedimientos clínicos o el control de la calidad asistencial, también deberemos ser capaces de enriquecer nuestros sistemas actuales con información específica de las enfermedades concretas que tratamos; por ejemplo, cáncer de mama, Cardiopatías, enfermedades isquémicas... Este enriquecimiento también nos capacitará para la creación de nuevos algoritmos para las decisiones clínicas o para mejorar los algoritmos existentes. Actualmente casi toda la información relativa a la evolución de una patología especifica está redactada en informes no estructurados, y si bien el análisis semántico puede ayudarnos a sacar información para su estructuración y explotación, se necesita un trabajo previo de normalización de la información que se escribe en los informes, incorporando los conocidos protocolos clínicos y otros sistemas de codificación. En el Hospital La fe llevamos unos años trabajando en diseñadores de formularios específicos de enfermedad, que se integran en los sistemas tradicionales incorporando información que ya está en los sistemas, pero de forma muy dirigida y específica de la enfermedad que se trata y con una interfaz muy sencilla para el clínico.

Los sistemas de información se conciben de un modo genérico para satisfacer las necesidades funcionales de todos los departamentos de salud y con poca capacidad de adaptación, sin embargo, la realidad es que la variabilidad de la práctica clínica existe y los sistemas de información deben reflejarla. Es por ello que los gestores de la sanidad se encuentran en la encrucijada de determinar cómo serán los sistemas de información en el futuro, sin perder de vista que sean más parametrizables, más sencillo de implantar, más robustos y más amigables en su interfaz con el usuario. Las decisiones que hoy se tomen serán las que sustenten la incorporación de los nuevos retos de la medicina moderna en la próxima década. 


\section{Modelos de atención sanitaria}

Antes de la pandemia, el modelo asistencial estaba centrado en la presencia del paciente en las consultas, y solo algunos profesionales con interés por la tecnología complementaban las consultas presenciales con seguimientos telefónicos y sistemas de comunicaciones electrónicas como correo electrónico o sistemas de mensajería instantánea (de texto, voz o incluso vídeo). Esta incipiente práctica, no estaba muy extendida, pues era selectivamente aplicada a determinados perfiles de pacientes con ciertas habilidades digitales. La realidad antes de la pandemia es que mucha de la comunicación entre el profesional y el paciente se realizaba con la aplicación Whatsapp por acuerdo mutuo de ambas partes, y siendo conocedores de las implicaciones en la privacidad de ambos.

Con la irrupción de la pandemia el modelo asistencial centrado en la presencia del paciente ha saltado por los aires, y en cuestión de semanas, debido a las restricciones de movilidad y aforos, se normalizó la necesidad de teletrabajo y se empezó a trabajar en procedimientos que facilitaran la teleasistencia clínica. Y los profesionales de IT tuvimos que garantizar unas condiciones mínimas de acceso remoto, partiendo de una tecnología en la que hacía años que no habíamos invertido. De modo que dos proyectos menores, como eran el teletrabajo y la teleasistencia por su difícil encaje en el modelo tradicional que dominaba el día a día de las operaciones del hospital, pasaron a ser de prioridad máxima y maduraron en pocos meses.

La pandemia nos ha traído un nuevo modelo, ampliamente divulgado por analistas y teóricos de la gestión sanitaria, y que pasa por ser menos presencial, basado en las tecnologías de internet, pero igualmente cercano y personalizado. Hace años que sabemos que tenemos que modernizar la sanidad, y podemos decir que en el Hospital La Fe venimos trabajando más de una década para poder atender las peticiones de nuestros pacientes cada día más jóvenes y más informados, y adaptando los nuevos procesos también a la población menos digital incluso anciana que también necesita atención.

Estos trabajos, que constituían programas piloto en diversos ámbitos han sido el embrión que ha facilitado la transformación digital que ahora fuerza el nuevo paradigma social que ha causado la pandemia. La atención a los pacientes con todo tipo de patologías distintas del covid-19 no podía quedar congelada, no se puede abandonar a un ciudadano cuya situación clínica requiere revisión y tratamiento. Así que pasado el pico de la primera ola (de casos covid) y atendiendo a las restricciones de acceso y movilidad en los centros sanitarios, tuvimos que implementar el nuevo modelo de atención no presencial.

Para dar cobertura al nuevo modelo de atención tuvimos que habilitar una plataforma de tele consulta, y para facilitar el teletrabajo aumentamos las licencias de acceso remoto, así como el caudal de la red de datos. Estos nuevos procedimientos de trabajo supusieron también la compra de webcams y de auriculares, y reforzar el soporte técnico, que alcanzó un pico de demanda que nos llegó a desbordar.

Mientras llega, una solución corporativa de videoconsulta, en el Hospital se diseñó e implementó íntegramente un sistema que incluye una plataforma de streaming de video con licencia de software libre y un módulo de desarrollo propio que se incrusta 
en la aplicación de citas e historia clínica. La decisión de abordar este desarrollo la basamos en la inmediatez. No había nada en el mercado que acoplase una solución de videoconferencia integrada en el sistema de práctica clínica habitual y debía garantizar la seguridad que una herramienta de este tipo requiere. La lección aprendida de esta experiencia es que ni todos los profesionales de la salud, ni todos los pacientes están preparados para la videoconferencia, y hay casos en los que, aun conjugándose el profesional y el paciente idóneos, el caso requiere una atención presencial. A fecha de hoy menos del $5 \%$ de las consultas que se realizan en el hospital son "no presenciales" y la expansión de esta práctica se vislumbra muy lenta.

Sin embargo, en mi opinión si conseguimos el objetivo del informe España digital $2025^{1}$ que garantiza la conectividad digital al 100\% de la población, el uso de la videoconsulta en el entorno rural puede ser la clave de un cambio en el modelo de atención sanitaria con un grandísimo impacto positivo en los pacientes.

Uno de los retos fundamentales al que nos hemos enfrentado es el de identificar y autentificar al paciente cuando establecemos contacto con él por un medio telemático. De pronto el teléfono móvil, un dato que se había recogido en la historia clínica sin ningún tipo de validación, se convirtió en la llave necesaria para comunicarnos con el paciente. De un día para otro, tuvimos que anular más de 7000 citas presenciales diarias, hemos mandado mensajes con resultados negativos de prueba de covid, citas para vacunación, y reprogramaciones o anulaciones de citas médicas. Afortunadamente, ya contábamos con una plataforma de envió de SMS de la Generalitat Valenciana que veníamos usando para el recordatorio de citas por lo que pudimos modelizar los procesos para absorber las nuevas necesidades de envíos. Hemos exprimido la herramienta de mensajería, por ejemplo, para localizar pacientes pediátricos con asma, advirtiéndoles que si acudían a urgencias se trajeran su máscara de inhalación, con el fin de proteger el contagio entre pacientes. O también por ejemplo para el envío de los resultados de covid negativo a los colegios de nuestro departamento porque al Hospital se le encomendó esta tarea.

En este aspecto debemos implementar mecanismos para encontrar una clave de identidad digital con la que garantizar la comunicación con el paciente. Con el actual estado del arte de estos mecanismos, el reto está a nuestro alcance, "si todo el mundo tiene una identidad digital para sus redes sociales, o sus relaciones con la banca, o plataformas de compra online, ¿porque no podemos utilizar esos medios para proveer una identidad digital en el sistema sanitario?".

Vuelvo otra vez a la cultura digital como palanca facilitadora de la transición al nuevo modelo. Los nativos digitales son los nacidos a partir de 2000; esta población se adaptará fácilmente, pero el gran escollo es que un $5 \%$ de nuestra población es mayor de 72 años y constituyen el $25 \%$ de los usuarios hospitalarios, y la gran mayoría de ellos, lejos del paradigma digital, resultan inhábiles o incluso analfabetos en el mundo digital. Es por eso que al diseñar aplicaciones de servicios públicos hay que hacerlas sencillas, intuitivas, y robustas, y siempre contar con los canales alternativos, los

\footnotetext{
${ }^{1}$ https://www.lamoncloa.gob.es/presidente/actividades/Documents/2020/230720Espa\%C3\%BlaDigital_2025.pdf
} 
tradicionales, necesarios para mantener incluida a toda la población en su derecho a la asistencia sanitaria.

En resumen, el futuro inmediato potencia las herramientas sanitarias en forma de portales de servicios que ofrecen la posibilidad de establecer un contacto más personal entre el ciudadano y el personal sanitario, y donde convergen las necesidades del paciente, del clínico y de los gestores de la sanidad.

Los teóricos de la gestión sanitaria hablan de un entorno totalmente integrado en la información y comunicación individual con el paciente, y la realidad es que vamos caminando hacia ese modelo lentamente, con el ritmo que nos marca la sociedad y la evolución de las personas que conforman los equipos clínicos y siempre dependiendo de las capacidades de los sistemas de información.

Es seguro, que el futuro de la sanidad pasa por el desarrollo de lo que hoy conocemos como "telealgo", y en ese sentido los responsables de Sanidad del Gobierno Valenciano están en fase de adjudicación de un pliego para el desarrollo de un software que dé respuesta a una plataforma de servicios digitales para la salud. Para el desarrollo de esta plataforma hay que tener en cuenta las necesidades del negocio y de nuestros pacientes, pero también se debe contar con la madurez en cultura tecnológica de los profesionales y de los usuarios de estos servicios, cuestión que en mi opinión es la clave del éxito de estos proyectos, para no quedarnos en un proyecto piloto. Si se dan estas condiciones podríamos decir que el desarrollo de nuevas plataformas digitales será un hecho y será la gran oportunidad para evolucionar hacia un nuevo modelo de atención sanitaria.

\section{La medicina moderna y la información: La toma de decisiones y la investigación}

Los próximos avances en tecnología auguran a los profesionales del sector sanitario un futuro apasionante. Incorporaciones al mundo de la sanidad como la inteligencia artificial, el manejo masivo de datos y algoritmos de predicción van a permitir nuevas pautas de tratamiento o análisis de enfermedades mucho más personalizadas. Asistiremos a una revolución en los dispositivos de atención al paciente: vendajes eléctricos, sondas inalámbricas, chips de control orientados a patologías concretas... El aparataje de intervención y la electromedicina también se ha subido al tren de la modernización: bisturís moleculares, intervenciones en remoto, robots quirúrgicos... Además, los equipos de imagen clínica en color que mejoran el contraste para un diagnóstico más preciso y la impresión en tres dimensiones que posibilitarán la generación de modelos para ensayar intervenciones, o la producción de implantes y órganos artificiales personalizados y adaptados a cada paciente.

Todos estos avances tienen por delante un gran recorrido hasta alcanzar un grado de madurez, de seguridad y calidad que permita su uso generalizado. Algunos de ellos serán una realidad y otros se descartarán, como siempre pasa en la incorporación de nuevas tecnologías. 


\section{Transformar la información en conocimiento}

La transformación de la información para convertirla en conocimiento es necesaria para la evolución hacia mejores diagnósticos, mejores tratamientos y mejores decisiones en el entorno sanitario, lo que revertirá en mejoras en la tecnología. El tiempo de la medicina de precisión ha llegado, el uso de la información como un nuevo recurso natural es necesario para conseguir la medicina personalizada que tanto interés despierta en nuestro entorno.

Tenemos que empezar por algo obvio, en el sistema nacional de salud existen muchos sistemas de información clínica pero el nivel de integración y coexistencia entre los mismos todavía es muy bajo. Para poder llegar a hacer un uso de la información clínica de una manera global, ya sea en diagnostico o investigación, es necesaria la creación de ecosistemas informáticos que aglutinen toda la información del paciente.

Este es el primer escalón para poder procesar automáticamente y de forma holística la información de un paciente en el tratamiento de su problema de salud, o bien para avanzar en la investigación de una patología con grandes conjuntos de datos relativos a grupos de individuos que la padecen. Y cuando tengamos estos pasos consolidados podremos incorporar nuevas fuentes de información como las redes sociales, apps en la nube, datos captados mediante sensores y otros dispositivos móviles de monitorización con conexión inalámbrica y toda la información de salud externa al hospital que ya hoy se está generando a veces sin ser conscientes de ello.

Mi propuesta relativa al uso y explotación de los datos es enfocar a las organizaciones sanitarias en dos aspectos: En primer lugar, ocuparnos de la "democratización" de la información para toda la organización y en segundo lugar conseguir el retorno de la información al profesional que la genera para la mejora de sus decisiones clínicas.

Para democratizar la información debemos generar sistemas que permitan poner a disposición de cualquier mando operativo de la empresa información para tomar sus decisiones y hacerlo en tiempo real. También hay que contextualizar la información, no es lo mismo el contexto de la gestión sanitaria que el contexto de la gestión operativa de un servicio; por ejemplo, obtener información de listas de espera quirúrgica cada seis meses para publicarlas en el portal de Sanidad es útil para la transparencia de la administración, pero esa misma información con ese decalaje en el tiempo es inútil para tomar decisiones para disminuir la lista de espera por parte del cirujano que la gestiona.

La base que mejora este aspecto ya está puesta, aunque la mayoría de los indicadores que se usan en salud se basan en información ya recopilada, modelizada e interpretada para tomar decisiones en el nivel estratégico, muy de gerencias de hospital o dirección general de administración sanitaria. Tenemos que modernizar la forma de acceso a la información, y sin perder lo alcanzado en el nivel de la estrategia sanitaria, escuchar a los servicios clínicos y sus mandos intermedios para enfocarnos en el nivel operativo. Este reto debemos abordarlo de manera inmediata para afinar en el uso de recursos en el funcionamiento diario de la atención que redundara en una mejora en la atención a nuestros pacientes. 
En cuanto al segundo aspecto, el retorno de la información para la mejora de las decisiones clínicas, hemos aceptado desde siempre que los profesionales introducen la información en los sistemas y después, respondiendo a una necesidad muy concreta, se puede hacer una petición para sacar algún tipo de información. Pero en general, el diseño de los sistemas no está devolviendo la información a los que la generan, y esto es un perjuicio directo que impide la toma de mejores decisiones clínicas.

La implementación de avisos, alertas o cualquier sistema de ayuda a la decisión integrados en el sistema de historia clínica será una obligación en los próximos años. Actualmente se está empezando a esbozar alertas que facilitan las decisiones como por ejemplo el cálculo de valores del cáncer de próstata con datos de diversos sistemas; o implementación de alertas basadas en escalas de gravedad al ingreso;

El desarrollo de estas capacidades son una deuda que los que dirigimos la estrategia de los sistemas de información tenemos con el usuario final: devolver en forma de conocimiento la información que introducen con el objetivo de simplificar y agilizar la toma de sus decisiones clínicas.

\section{Big Data. Uso masivo de datos para la investigación}

Como no, en este punto debemos hablar del bigdata. Desde hace unos años hemos abierto ese camino en el campo sanitario, donde ya contamos con una gran cantidad de datos, hablamos de millones de registros almacenados en múltiples formatos, y con la capacidad de herramientas software aplicables en este campo para el proceso de grandes volúmenes de información.

La historia clínica electrónica se compone de datos estructurados con valores numéricos o texto, imágenes, gráficas, ficheros de texto, y otros formatos, y ya tenemos el embrión de la medicina personalizada gracias a la conjugación de todos estos datos recopilados de miles de hospitales que proporcionarán información de millones de pacientes para alimentar nuevos algoritmos de inteligencia artificial basados en métodos predictivos y en reconocimiento de lenguaje natural. Así, el bigdata es otra pieza que se incorpora al tablero de juego en el campo de la investigación en salud.

Mas allá de la atención de los problemas de salud de los ciudadanos, en el modelo sanitario actual la investigación es una parte importante del desempeño de nuestros profesionales. Los resultados de la investigación revierten en la calidad asistencial y en el éxito de los casos clínicos, y por tanto los gestores de la sanidad pública deben ponerla en sus planes estratégicos, porque no se concibe la sanidad sin investigación. Lo que vemos en el hospital La Fe, es que hay muchos proyectos que no tienen un acceso directo a los datos, y esto es así porque aún no se han sentado las bases. No se ha hecho aún en la organización una definición de la estructura de Big Data, ni se ha dotado de una infraestructura estándar, abierta y disponible para todos los profesionales y grupos de investigación.

Es en este campo de la investigación donde son más necesarios los cambios en cuanto a uso de datos en los próximos años. Por eso, son muy importantes las 
políticas que fomenten la compartición de datos de salud tanto a nivel nacional, como en el seno de la Unión Europea. Afortunadamente, el cambio de milenio ha traído iniciativas legislativas europeas y nacionales que regulan la libre circulación de datos entre estados de la unión, o la reutilización de la información en el sector público, y con ellas programas plurianuales de desarrollo de la mayoría de los sectores estratégicos, con importantes aportes económicos, por ejemplo, el programa europeo conocido como horizonte $2020^{2}$.

El estímulo económico europeo y nacional ha facilitado las iniciativas hacia los objetivos de open data, promovidas por algunos grupos de investigación en los grandes hospitales españoles, y algunas muestras podemos verlas desarrolladas a través del Instituto de Investigación Sanitaria La Fe, por iniciativa propia, o en consorcio con otros hospitales nacionales o europeos. Yo veo un importante reto para los servicios encargados de los sistemas de información, aprovechar estas políticas y estos programas para desarrollar o adquirir herramientas software que permitan conocer las necesidades de información de los proyectos de investigación. Esto dotará la capacidad de presentar la información en la cantidad precisa, con las características necesarias y los formatos adecuados. De la capacidad que tengamos de crear sistemas conjuntos o normalizados y accesibles para el mayor número de investigadores dependerá nuestro éxito. La pandemia ha evidenciado ante los ciudadanos la necesidad de investigar bien y rápido, y eso solo es posible mediante la colaboración entre sistemas sanitarios nacionales y europeos.

En resumen, es necesario la normalización de la información a compartir, crear repositorios de datos accesibles, definir los estándares de intercambio, promover la colaboración entre administraciones sanitarias, y disponer de capacidades para la anonimización de la información. Y esto hará que la investigación en salud sea un gran activo a nivel regional, nacional y europeo.

\section{Ciberseguridad, privacidad de la información, y cumplimiento normativo}

Es el momento de hablar de seguridad. Siguiendo el hilo iniciado en la introducción, puesto que cualquier acto clínico se sustenta en las tecnologías de la información, en todo momento las personas que ejercen el rol clínico deben tenerla disponible en su puesto de trabajo. Para no errar en el diagnóstico la información clínica debe ser veraz y permanecer íntegra; y cuando ponemos al sujeto paciente en el centro de la atención, se evidencia el deber de salvaguardar su derecho a la intimidad y por tanto el carácter confidencial de la información que se maneja.

En este ámbito se plantean dos líneas de actuación, la primera sería la de la protección de los sistemas, y la segunda la de la protección del individuo. Y para ambas líneas el legislador nos aporta dos herramientas de trabajo: la primera consiste en las regulaciones sobre seguridad de los sistemas en administraciones públicas y otras sobre infraestructuras críticas ${ }^{3}$, y para la segunda tenemos las leyes sectoriales que

\footnotetext{
2 https://ec.europa.eu/programmes/horizon2020/en

${ }^{3}$ Fundamentalmente el Real Decreto 3/2010 de 8 de enero, que regula el Esquema Nacional de Seguridad, la Ley $8 / 2011$, de 28 de abril, por la que se establecen medidas para la protección de las infraestructuras críticas, su reglamento en el Real Decreto 704/2011, de 20 de mayo. Y el Real Decreto-ley 12/2018, de 7 de septiembre, de seguridad de las redes y sistemas de información.
} 
regulan la autonomía del paciente, la documentación clínica o la investigación ${ }^{4}$, así como las leyes generales sobre protección de datos 5 .

\section{Preservar la ciberseguridad}

Desde 2017 los analistas informan cada vez más de la tendencia creciente de ataques dirigidos contra equipos médicos con intención de extorsionar, robar información o afectar al servicio, mediante el uso de malware de cualquier tipo: Un equipo infectado puede dejar de funcionar e interrumpir la atención médica que presta, y esto predispone a las víctimas a pagar un rescate para evitarlo. Otro interés del delincuente es el robo de información clínica para ponerla a la venta en la dark web.

Según el informe 2020 "Ciberamenazas y tendencias" del Centro Criptológico Nacional, la pandemia de la COVID-19 ha propiciado la aparición de amenazas para la seguridad de la información por la sobreexposición pública de los sistemas informáticos de las empresas, debido a las medidas de confinamiento adoptadas, y el consecuente despliegue masivo de entornos tecnológicos de teletrabajo (sistemas de acceso remoto, servicios de videconferencia, servicios colaborativos en la nube ...). La mayoría de equipamiento médico está conectado a las redes de ordenadores, y desde 2019 se observa una tendencia creciente de ataques que utilizan las debilidades de estos sistemas y diversos tipos de malware a muchas infraestructuras y servicios sanitarios.

Las administraciones sanitarias, conscientes de las ciberamenazas en su entorno, ya exigen a sus proveedores de tecnología la incorporación de medidas técnicas en los equipos que compran para prevenirlas y preservar la privacidad de la información que generan. Y los fabricantes cada vez se ocupan más de priorizar la implementación de controles de seguridad y medios para parchear los equipos contra las vulnerabilidades que aparezcan.

La necesidad causada por la pandemia de dar una respuesta no presencial a las necesidades asistenciales se ha resuelto abriendo nuevas aplicaciones de servicios virtuales en internet, dejando nuestros datos personales mucho más expuestos que antes. Como ejemplo, hemos podido ver una prueba de concepto en la que un hacker se cuela en una consulta clínica sin controles de acceso, pincha un equipo a la red local, que está desprotegida, accede al PACS, descarga una imagen médica de un paciente, y la modifica añadiéndole información para que se vea un tumor donde no lo hay. Obviamente, los centros sanitarios ponemos nuestras medidas de seguridad físicas e informáticas para evitarlo, pero la posibilidad existe, y la administración sanitaria debe preocuparse de analizar todos los riesgos, y poner los medios para eliminarlos. El anterior es un ejemplo que puede ocurrir en una red local, pero hay

\footnotetext{
${ }^{4}$ Fundamentalmente la Ley 41/2002, de 14 de noviembre, básica reguladora de la autonomía del paciente y de derechos y obligaciones en materia de información y documentación clínica, y la Ley 14/2007, de 3 de julio, de Investigación biomédica

${ }^{5}$ Fundamentalmente el Reglamento (UE) 2016/679 del Parlamento Europeo y del Consejo, de 27 de abril de 2016, relativo a la protección de las personas físicas en lo que respecta al tratamiento de datos personales y a la libre circulación de estos datos y por el que se deroga la Directiva 95/46/CE y nuestra Ley Orgánica 3/2018, de 5 de diciembre, de Protección de Datos Personales y garantía de los derechos digitales.
} 
más, ¿qué nos puede ocurrir si conectamos equipos a internet? relojes inteligentes con aplicaciones hackeadas que medirán mal el ritmo cardíaco, marcapasos hackeables por bluetooth, puertas traseras que dan acceso a las máquinas de radioterapia y cualquier otro que pueda abarcar la imaginación.

Es por ello que en el entorno sanitario en los próximos años tenemos que integrar, promover y exigir la seguridad en todos nuestros procesos asistenciales y sistemas críticos, haciendo que la seguridad sea un requerimiento obligatorio en los dispositivos que adquiramos desde el momento de su diseño.

\section{Preservar la privacidad de las personas}

La normativa en protección de datos obliga a las organizaciones a documentar en un registro los tratamientos de información personal, indicando la procedencia de los datos, identificando quien es el responsable del tratamiento, cual es la finalidad del tratamiento y su legitimación; y obliga a informar a la persona afectada, quien debe conocer cuáles son sus derechos, como ejercerlos, si hay subcontratistas encargados del tratamiento u otros terceros destinatarios de su información personal, y regula perfectamente las condiciones para las transferencias transfronterizas (las realizadas entre estados en el ámbito de la unión europea) o las transferencias internacionales de información (las realizadas fuera del ámbito de la Unión Europea).

En 2018 entró en vigor el reglamento europeo de protección de datos, y el hospital la fe puso al día su gestión de la seguridad y la privacidad de acuerdo con él. Implementando las medidas requeridas, como el registro de actividades de tratamiento de datos, los procesos de ejercicio de derechos del paciente relativos a la protección de datos, el procedimiento de registro y declaración de brechas de seguridad etc.

\section{Conclusiones}

Los modelos de asistencia sanitaria son dinámicos, han cambiado y seguirán haciéndolo, el modo de contacto entre el paciente y el profesional está evolucionando, haciéndose cada vez más telemático y para conseguirlo la medicina necesita modernizar sus herramientas.

Si buscamos una conclusión desde una perspectiva realista, queda claro que, para adoptar los nuevos modelos sanitarios, es necesario avanzar más deprisa en tecnología de lo que lo hemos hecho en los últimos años si queremos cambiar a nuevos modelos sanitarios.

Aunque los nuevos avances tecnológicos tardan décadas en llegar a la totalidad del sistema, una situación de emergencia, como esta pandemia en la que nos encontramos inmersos, ha hecho que se reduzcan los tiempos de implantación. En cualquier caso, el diseño del nuevo sistema se debe enfocar tanto en el paciente como en el profesional, por lo que tendremos que escuchar y atender las necesidades de nuestros pacientes, pero sin perder de vista la realidad de nuestros profesionales. 
Los pacientes y los profesionales de la salud necesitan desarrollar nuevas capacidades digitales y a su vez la implementación de los nuevos sistemas requiere una dotación suficiente de ingenieros de las distintas ramas tecnológicas involucradas en el nuevo paradigma asistencial.

La implementación de un sistema seguro, y la protección de los sistemas que ya funcionan en todas las áreas de la prestación sanitaria, va a necesitar muchos más recursos, no perdamos de vista que durante la pandemia han quedado abiertas las puertas del hospital a Internet y no debemos ser ajenos a los riesgos que estamos expuestos cuando nos conectamos a la red de redes mundial.

Por último, considero que hay que retocar la estructura de la administración sanitaria para poder cambiar el ritmo de avance de estos nuevos modelos. Y es responsabilidad de las personas que ostentan el poder en el gobierno de la nación y autonómico apostar por una sanidad moderna para los ciudadanos, invirtiendo en personal, equipamiento y tomando las decisiones acertadas y orientadas al bien común. 